\title{
Cardiovascular Changes in Cardiogenic and Obstructive Shocks: Analysis Using a Cardiopulmonary Simulation Model
}

\author{
M Giannessi ${ }^{1}$, NW Chbat $^{2}$, A Albanese $^{1}$, J Op Den Buijs ${ }^{3}$, \\ E Magosso $^{1}, \mathrm{M} \mathrm{Ursino}^{1}$ \\ ${ }^{1}$ University of Bologna, Cesena, Italy \\ ${ }^{2}$ Philips Research North America, Briarcliff Manor, NY, USA \\ ${ }^{3}$ Mayo Clinic, Rochester, MN, USA
}

\begin{abstract}
Conditions characterized by a fall in cardiac output are associated with alterations in cardiopulmonary variables and activation of cardiovascular and respiratory control mechanisms. In order to study these complex relationships, we developed a comprehensive cardiopulmonary model consisting of a circulation, a respiration and a metabolism block. In this work, the model is used to simulate cardiovascular dynamics in pathological conditions with an acute decrease in heart function. In particular, two conditions are examined: the first is characterized by a decrease in heart contractility, simulated in the model via a reduction in the left ventricular end-systolic elastance. The second consists in an increase in pulmonary arterial resistance, associated with pulmonary embolism. In conclusion, the proposed model may be of value in clinical settings to illustrate the complex relationship among cardiovascular variables.
\end{abstract}

\section{Introduction}

Conditions characterized by a fall in cardiac output (CO) represent a frequent cause of mortality and morbidity, while their rapid diagnosis and efficient treatment still represent a demanding task for physicians. Shock, or acute hypotension, that accompanies this fall is detectable in an intensive care unit (ICU) scenario. However, shock can be caused by heart failure, or by blood obstruction, loss, or infection. These four different causes require very different therapeutic routes. Gaining insight on the different shock types becomes then clinically important. In this paper, we present simulation results and analysis for cardiogenic shock (due to heart failure) and compare it with published patient data, and for obstructive shock (due to pulmonary emboli).

A reduction in $\mathrm{CO}$ induces a fall in systemic arterial pressure and in oxygen transport, thus triggering regulatory actions. Arterial hypotension activates the baroreflex response, which causes an increase in sympathetic drive and a reduction in vagal tone to the heart, with consequent increase in total peripheral resistance and heart rate (HR), as well as venoconstriction. The fall in oxygen delivery, in turn, may stimulate local regulatory mechanisms, able to vasodilate coronary and brain circulations. In case of severe heart failure resulting in arterial hypoxia, other mechanisms can be put into action, such as the arterial chemoreflex and the lung-stretch receptor mechanism (consequent on respiratory changes). Of course, these mechanisms interact in different ways, depending on the severity of the cardiac insufficiency, on its aetiology and on differences of the individuals.

The complexity of the relationships involved in shock and the role of individual parameter changes, can be better understood with the use of mathematical models and computer simulations. In previous years, we developed a comprehensive mathematical model of systemic and pulmonary circulation integrated by the short-term cardiorespiratory regulation [1]. The model is able to simulate cardiovascular changes in different acute stresses (like haemorrhage, hypoxia and hypercapnia) $[1,2]$.

In the last months, the previous work was further developed to: i) include respiratory mechanics and metabolic exchange; ii) use the extended model to simulate conditions of clinical relevance in the ICU.

In the present work, the model is used to analyze the cardiovascular changes occurring during two different conditions leading to reduced cardiac efficiency: a reduction in cardiac contractility (simulated decrease of the end-systolic elastance of the left ventricle), and a reduction in left-ventricle filling (simulated increase of the pulmonary arterial resistance). Results illustrate the correlation of the main cardiovascular changes with the level of myocardial dysfunction and the size of the pulmonary embolus. 


\section{Methods}

The model includes four cardiac chambers with ventricular contractility, pulmonary and systemic circulations (see Fig. 1). The latter is subdivided into five districts arranged in parallel (brain, coronary, skeletal muscle, splanchnic and the remaining extrasplanchnic vascular beds).

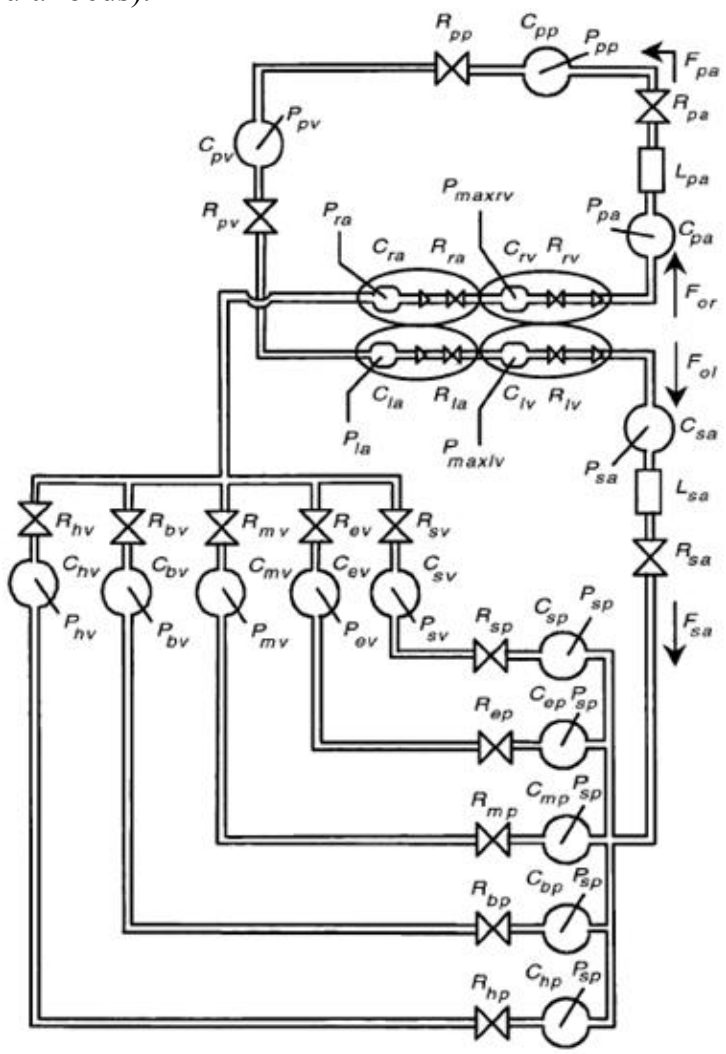

Figure 1. Hydraulic analog of the model in which resistances are represented with restrictions and compliances with expanded spaces. P, pressures; R, hydraulic resistances; C, compliances; L, inertances; F, flows; sa, systemic arteries; sp and sv, splanchnic peripheral and splanchnic venous circulation; ep and ev, extrasplanchnic peripheral and extrasplanchnic venous circulation; $\mathrm{mp}$ and $\mathrm{mv}$, peripheral and venous circulation in the skeletal muscle vascular bed; bp and bv, peripheral and venous circulation in the brain; hp and hv, peripheral and venous circulation in the heart (coronary vascular bed); la, left atrium; lv, left ventricle; pa, pulmonary arteries; $p p$ and pv, pulmonary peripheral and pulmonary venous circulation; ra, right atrium; rv, right ventricle.

According to Fig. 1, the different compartments include hemodynamics in the large arteries (systemic and pulmonary), the peripheral circulation, and the venous circulation. Three main kinds of lumped parameters are used in building the model: inertances, which account for inertial forces in blood (these are relevant in large arteries only); resistances, which account for pressure energy losses (this parameter is especially important in the peripheral circulation) and capacitances, which represent the blood volume stored in each compartment (this parameter is especially relevant in the venous circulation). Volume, in turn, is divided in an unstressed volume, which does not stretch the vessel wall, and a stressed volume (which causes stress in the wall, and hence is associated with increase in internal pressure). The reader can find more details, equations and parameter values in former publications $[1,2]$.

Short term regulatory mechanisms work via local metabolic factors on peripheral resistances and through the action of the autonomic nervous system. The latter modulates several parameters via two major efferent neural branches, i.e., the sympathoadrenergic and the vagus. Vagal stimulation rises heart period. Sympathetic stimulation causes vasoconstriction in arterioles and veins (thus increasing peripheral resistances and decreasing venous volumes), raises heart contractility, and increases heart rate. Some vascular beds (splanchnic circulation, skeletal muscle circulation and circulation in the other extrasplanchnic districts) are very sensitive to sympathetic stimulation. Conversely, the cerebral and coronary circulations are independent of the autonomic control mechanism. Finally, the sympathetic and vagal neural branches, respond to several groups of afferent influences, triggered by baroreceptors, chemoreceptors and lung-stretch receptors.

The cardiopulmonary model (see Fig. 2) also includes lung mechanics (with distinction among larynx, trachea, bronchi and alveoli), metabolic exchange equations at the alveoli and capillaries, and the control of respiratory rate and tidal volume by central and peripheral chemoreceptors.

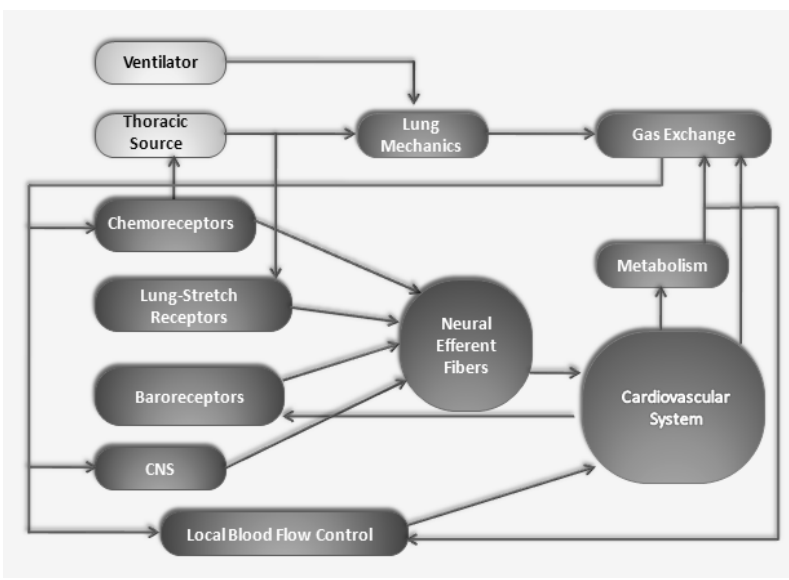

Figure 2. Cardiopulmonary model diagram. 


\section{Results}

Though the model represents cardiopulmonary dynamics with associated neural regulatory mechanisms, here we are only perturbing the cardiovascular part of the model and analyzing its response. Let us first clarify the convention that variables means blood pressures, flows and volumes, and parameters pertains to blood vessel properties and geometry, i.e. the $\mathrm{R}, \mathrm{L}$, and $\mathrm{C}$ of the different compartments defined earlier. The two shock scenarios that were simulated refer to acute conditions, and acute response only is what we analyze.

The first scenario is one of a cardiogenic shock. Please refer to Fig. 3 for abbreviations of variables and results. Six variables are shown which are important in assessing the condition of an ICU patient in such a shock (acute hypotension). The cardiopulmonary model has parameters that would correspond to a $70 \mathrm{Kg}$ male with initial variables in the quasi-normal range (EF 55\%, CO $5.3 \mathrm{~L} / \mathrm{min}, \mathrm{HR} 72$ beats $/ \mathrm{min}$, and slightly elevated systolic/diastolic 128/87 and PCWP $15 \mathrm{mmHg}$ ) and so representing relatively stable patient conditions. The parameters of the model may be changed manually or automatically to represent different classes of patients or a particular patient with changing conditions. In this study, however, the parameters are held constant as to represent a general nominal ICU population.
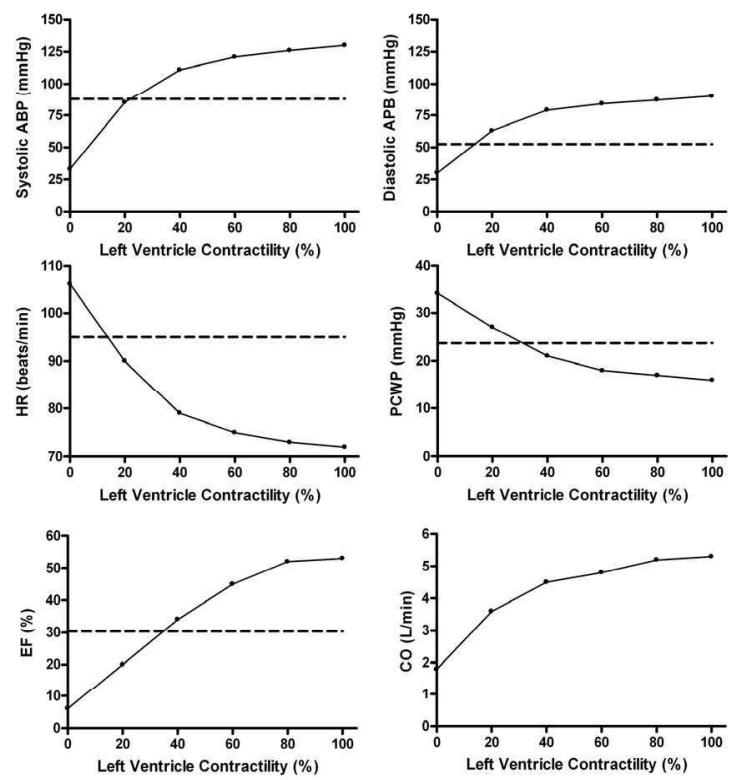

Figure 3. Plots of systolic arterial blood pressure (systolic $\mathrm{ABP}$ ), diastolic arterial blood pressure (diastolic ABP), heart rate (HR), pulmonary capillary wedge pressure (PCWP), ejection fraction (EF) and cardiac output (CO) vs the contractility of the left ventricle, computed by the model at steady state conditions by performing several step changes to the end-systolic elastance. Model results (continuous line with dots) are compared with data from Webb JG. et al. [3] (dashed line). The dashed lines represent mean values of the different variables monitored for 816 patients suffering cardiogenic shock.

The results show that as the left ventricular (LV) elastance worsens, blood pressure and corresponding $\mathrm{CO}$ and EF fall precipitously, while the PCWP increases due to blood backing up all the way to the pulmonary circulation. HR also increases, which includes baroreflex action trying to compensate for arterial blood pressure drop. With a further deterioration of the LV contractility, PCWP enters the $20 \mathrm{mmHg}$ zone, which could cause interstitial edema to be likely followed by pulmonary edema. It is intuitively helpful to correlate the level of LV contractility loss to corresponding values of the PCWP, $\mathrm{EF}$, and $\mathrm{CO}$. No $\mathrm{CO}$ results were reported in the patient study. The simulation results fall well within the range of the population study. However, this real patient study has only averaged values for all five variables shown in dashed lines. According to the model, the patient population had an average of more than $50 \%$ loss in LV contractility. This loss could also be interpreted as equivalent loss of contractility as other factors could have contributed to the deterioration of these patients. A population breakdown would have been helpful to assess the severity of myocardial infarcts, comorbidities, demographic information, etc.

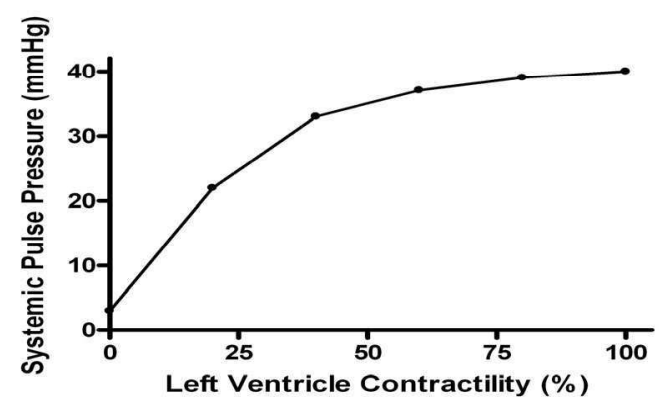

Figure 4. Systemic pulse pressure vs contractility of the left ventricle, computed with the model in steady state conditions by performing several step changes to the endsystolic left ventricular elastance.

Figure 4 represents the pulse pressure, the difference between systolic and diastolic pressures. Because the contractility of the left ventricle is affected, then the endsystolic pressure and volume will worsen more than the end-diastolic pressure and volume. Hence, the pulse pressure, and EF, as expected, will fall. No pulse pressure data was given in the patient study; however, a difference between the two dashed lines of the systolic and diastolic pressures of Fig. 3 would give an approximation of the 
patients' average pulse pressure value: around $30 \mathrm{mmHg}$.
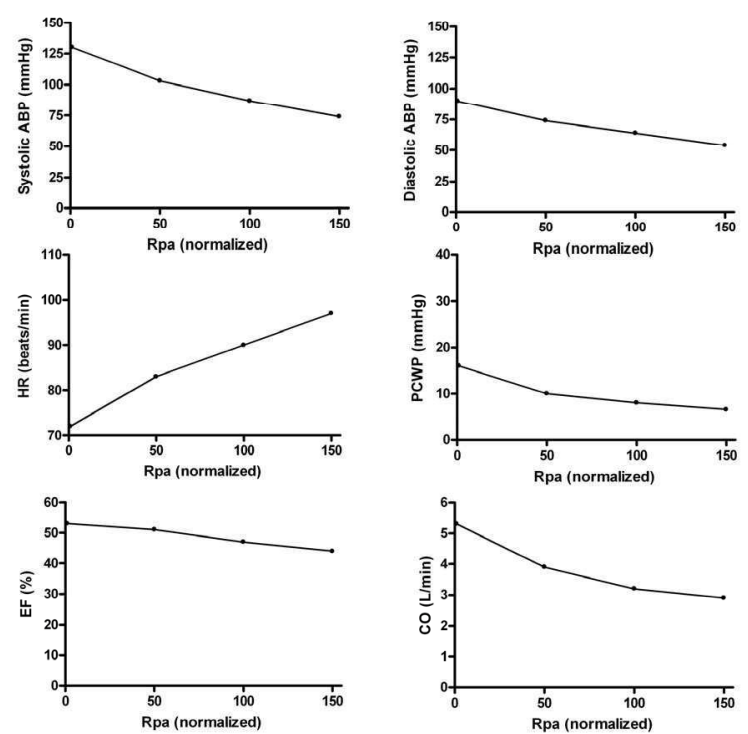

Figure 5. Plots of the main cardiovascular variables vs pulmonary arterial resistance (Rpa), computed with the model in steady state conditions by increasing 50, 100 and 150 times the Rpa. Rpa values are normalized at the basal value. Meaning of symbols is the same as in Fig. 3.

The second scenario is one of an obstructive shock, where the pulmonary artery resistance (Rpa) is acutely increased. An example of this condition would be a "saddle embolus", i.e. clogging of both pulmonary arteries, while a lesser severity shock would block a correspondingly lesser area of these arteries. The diameter of the pulmonary artery is implicit in the resistance (resistance is inversely proportional to the fourth power of the diameter.) In this shock the acute hypotension is not due to a malfunction of the left heart, as in the previous simulated scenario. So we see from the results in Fig. 5 that the EF falls only to $45 \%$ even after $150 \%$ increase in Rpa, while the CO falls much more (5.3 to $3 \mathrm{~L} / \mathrm{min}$ ), and so does blood pressure, due to the fact that less blood is reaching the left heart. HR also increases here in order to compensate for the hypotensive condition. PCWP falls since the obstruction occurs upstream from it, and hence less blood reaches the pulmonary capillaries. There were no patient results available in the literature for this scenario. However, a search is underway to obtain more patient data.

\section{Discussion and conclusions}

The presented results demonstrate that the model is able to simulate the main acute cardiovascular changes due to cardiogenic and obstructive shocks quite well. These include a fall in systolic, diastolic, and pulse pressures, a reduction in $\mathrm{CO}$ and $\mathrm{EF}$, and an increase in heart rate up to $100-110$ beats/min. PCWP increases with worsening LV contractility but decreases with increasing upstream pulmonary embolism. These changes make physiological and clinical sense.

The cardiogenic shock results are further compared (Fig. 3) with average values taken from a large data set of cardiogenic shock patients [3]. This comparison shows that the model can simulate the average cardiovascular variables, not only in a qualitative but also in a quantitative manner, assuming that the end-systolic elastance is decreased to $1 / 4$ or $1 / 5$ of its normal value. The results provide a quantitative indication of the degree of ventricular contractility impairment, which can occur in a typical cardiogenic shock patient, as a function of important hemodynamic variables such as $\mathrm{CO}, \mathrm{EF}$, and PCWP.

The present simulations describe only acute events with corresponding short-term hemodynamic response at different levels of parameter derangement (either in systolic elastance or Rpa). However, long-term shock effects which can progressively evolve with time and involve a downstream spiral, in which ischemia could progressively deteriorates heart function, which in turn further worsens ischemia [4] may be attempted in future work.

The present model can have various potential clinical applications in cardiology by providing insight into: interactions of cardiovascular variables (pressures, flows, volumes), effect of parameters (vessel properties and geometry) on hemodynamic stability, patient variability, correlation of parameter impairment severity with crucial cardiovascular variables, and finally possible therapeutic what-if scenarios.

\section{References}

[1] Ursino M, Magosso E, Acute cardiovascular response to isocapnic hypoxia. I. A mathematical model, Am. J. Physiol Heart Circ. Physiol, vol. 279, no. 1, p. H149-H165, July2000.

[2] Magosso E, Ursino M, A mathematical model of $\mathrm{CO} 2$ effect on cardiovascular regulation, Am. J. Physiol Heart Circ. Physiol, vol. 281, no. 5, p. H2036-H2052, Nov.2001.

[3] Webb JG, Kelly RF, Epidemiology of Cardiogenic Shock. In: Hollenberg SM and Bates ER. Cardiogenic Shock. United Kingdom: Blackwell Futura, 2002: chapter 1.

[4] Hollenber SM, Cardiogenic shock, Crit Care Med. 17: 391410, 2001.

Address for correspondence:

Massimo Giannessi

via Venezia 52, 47023 Cesena (FC), Italy

massimo.giannessi@unibo.it 